\title{
A DISCUSSION OF THE FIRST LAW OF THERMODYNAMICS FOR OPEN SYSTEMS
}

\author{
by H. A. TOLHOEK and S. R. DE GROOT
}

Instituut voor theoretische Natuurkunde der Universiteit, Utrecht, Nederland

\section{Synopsis}

In this paper it is discussed to what extent the first law of thermodynamics is a physical law, and to what extent it is a definition, if it is generalized so as to be valid for open systems. The conclusion is reached that it is partly a definition. Different generalizations of the first law, which can be proposed, must be considered as different definitions. The usefulness of these different definitions is compared with respect to the shift of arbitrary constants in the internal energy and in view of the thermodynamics of irreversible processes.

$\S 1$. Introduction and results. While $\mathrm{G} \mathrm{i} \mathrm{b}$ b s' form of the second law of thermodynamics for open systems is extensively employed, relatively little is found in the literature on a consistent use of the first law for open systems. This may be due to the incomplete physical interpretation of the equations which have been proposed for this law. In this paper we try to give a contribution to the problem of the clarification of the physical meaning of the first law for open systems, in particular about the problem as to how far it is a physical law and how far it is a definition.

The generalization of the first law for closed systems, to a law valid for open systems as well, is usually achieved in either of two ways for which the formalism has been given before $\left.{ }^{1}\right){ }^{2}$ ) (cf. also $\left.{ }^{3}\right)$ ). From the discussion of these formulations, (3) and (9), we find in $\S 2$ that the first law for open systems is a physical law only if no diffusion phenomena occur. In that case the formulae (3) and (9) are identical. In the general case with diffusion, however, the formulae become different, and more or less convenient, definitions of the heat transfer.

The convenience of the forms (3) and (9) is discussed in $\S 3$ with 
respect to the shifting of the arbitrary zero point in the partial specific energies. It is found that the heat transfer defined by (3) is affected by these shifts, while the heat transfer of (9) is not changed (just as in the first law for closed systems).

In the discussion on the physical meaning, the usual forms of the first law, valid for uniform systems, were employed for the sake of simplicity. All conclusions are, however, valid for non-uniform systems as well. The forms of the first law, containing specific quantities as required for non-uniform systems, are given in $\S 4$.

Finally, the effect of the two alternative forms of the first law for open systems on the form of the fundamental equations of macroscopic physics, as used in the "thermodynamics of irreversible processes" $\left.{ }^{1}\right)^{3}$ ), is summarized in $\S 5$.

§2. The first law for open systems. We write the first law of thermodynamics for closed systems in the form

$$
d U=d Q-p d V .
$$

With closed systems we mean systems that cannot exchange matter with their surroundings, though they may exchange heat with the surroundings. ( $p$ is the pressure which is exerted on the system, $V$ is the volume of the system). In addition to the term - $p d V$ there may be other terms, if other forces are acting on the system. We shall omit these terms in $\S \S 2-4$, since they have no importance for this discussion. The equation (1) expresses a physical law in the following sense : the quantities $p, V$ and $d Q$ can be measured; $p$ is a mechanical quantity and $V$ a geometrical quantity. The heat $d Q$ supplied to the system by the surroundings can be measured, if we think of these surroundings as a calorimeter, by measuring the temperature changes in the surroundings resulting from the heat flow into the system (we suppose the specific heats of the surrounding bodies, which change in temperature, to be known). Now it is observed that a quantity $d U$ defined by (1) is the differential of a quantity $U$ which is a function of the "state variables" characterizing the system. These "state variables" may be taken as volume and temperature for a homogeneous system with one component; as volume, temperature, and concentrations for a homogeneous system with several components. In this way the energy $U$ is defined as function of these "state variables". $U$ contains an arbitrary constant since (1) 
determines only the differential $d U$ and not $U$ itself. We now make the following convention:

The energies of different homogeneous systems of the same pressure, temperature and constitution are proportional to the masses of the systems.

This makes the energy an extensive quantity. The arbitrariness with which the energy can be changed by a constant is diminished by (2).

We now write down a generalization of (1) for open systems, i.e., for the case that the system may exchange matter with the surroundings; we suppose, however, that the system is homogeneous and that the matter flowing into or from the system has the same constitution as the matter in the system (hence this flow is a motion of the bulk of this matter without diffusion phenomena). We now put (1) p. 46)

$$
d U=d Q-p d V+h d M,
$$

where $h=(U+p V) / M$ is the specific enthalpy and $M$ is the mass of the system. In the equation (3) $d U$ is known according to (1) if the change of the state variables of the system is known. If the total mass of the system changes, (2) is also needed to fix the energy change $d U$, (we suppose that concentrations remain the same). The measurement of $p, d V$ and $d M$ involves no special difficulties and $h=(U+p V) / M$ being a state variable, is determined according to (1). However, we must be careful with the measurement of $d Q:$ as long as the heat transfer $d Q$ occurs through fixed walls it can be measured in the same way as for (1). If, however, a temperature gradient exists within the matter flowing from the system (fig. 1) the procedure for measuring $d Q$ can no longer be defined in the same way.

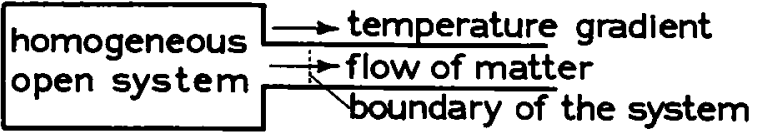

Fig. 1. For open systems a flow of matter may be accompanied by a flow of heat. It is not always possible to give a method to measure this flow of heat.

We may now define $d Q$ as the heat transfer, which is observed in matter of the same constitution which is at rest and in which the same temperature gradient exists. This means, that while we deter- 
mined $d Q$ for (1) by measuring the temperature change and heat capacity of the surroundings, $d Q$ in (3) is determined by measuring the temperature gradient and the heat conduction of the matter at the boundary of the system. We can do this only if no diffusion phenomena occur, as we have already supposed. With these suppositions all quantities in (3) can be measured, hence (3) can be considered as a physical law.

If we now take the case that diffusion phenomena occur in the matter flowing from or into the system, we can no longer specify a way to measure the heat transfer. The diffusion may be accompanied by heat flows (Dufour effect) which -are, however, not directly measurable. It is impossible to take the heat flow equal to the heat flow for the matter at rest, for, if diffusion occurs, coordinates can be chosen to make the bulk motion equal to zero, but the different components will still move in such a coordinate system.

In many cases it is still convenient to speak about the heat transfer $d Q$ even if flows of material with diffusion occur. This can be done by taking (3) as a definition of $d Q$ for such changes; all quantities can be measured except $d Q$; hence (3) cannot be verified (or falsified) for such changes, but the equation is sufficient to define $d Q$. If we state that $d U$ is defined for these changes, we must be careful: (2) fixes the ratio of the energy constants for several systems only if they have the same constitution; however, if diffusion occurs the constitution becomes different. Here we again fix energy constants by a convention which is a natural generalization of (2):

We take the energy of a system consisting of several subsystems to be the sum of the energies of the subsystems, it they are considered separately. (by doing this we suppose that the interaction amongst the subsystems is negligeable).

We apply this to find the way in which the energy of a mixture of components is fixed, once we have fixed the energies of the pure components. To this end we consider a system consisting of a volume containing a homogeneous mixture of a number of components, separated by a semipermeable wall from a volume filled with the pure component $k$, which can be brought into the main volume in a reversible way by moving a piston (fig. 2). As we could extract any component of a mixture in this way, we see that the energy constant of a mixture is determined, once the energy constants of the pure components are fixed. 
The partial specific energy $u_{k}$ of component $k$ in a mixture is defined by $u_{k}=\left(\partial U / \partial M_{k}\right)_{p, T}\left(M_{k}\right.$ is the mass of component $k$; $k=1, \ldots, n ; n$ is the number of components). Let $u_{k}^{p}$ be the specifc energy of the pure component $k$. We may change such a specific energy by a constant (independent of pressure and temperature) $\varepsilon_{k}$

$$
\tilde{u}_{k}^{p}=u_{k}^{p}+\varepsilon_{k} \text {. }
$$

From the situation in Fig. 2 and the definition of $u_{k}$ it follows immediately that $u_{k}$ must then be shifted by the same amount

$$
\tilde{u}_{k}=u_{k}+\varepsilon_{k},
$$

while the shift of the total energy

$$
U=\sum_{k=1}^{n} M_{k} u_{k}
$$

is given by

$$
\tilde{U}=U+\sum_{k=1}^{n} \varepsilon_{k} M_{k} \text {. }
$$

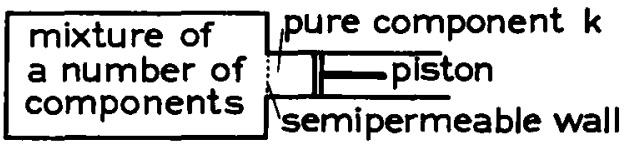

Fig. 2. If we have a mixture of several components, the energy shift of the mixture is related to the energy shifts of the pure components. This can be established if we remove some component through a semipermeable wall.

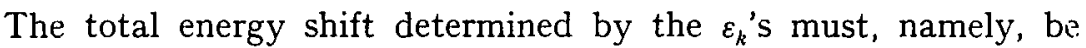
independent of the position of the piston since a different position changes only state variables of the system, while the same matter remains in the system (the complete system is "closed"). Hence, if we use (3) as a definition for $d Q$ for changes, where matter flows with diffusion occur, a certain abitrariness may occur as a consequence of the fact that the partial specific energies may be shifted. Since (3) must be considered only as a definition for changes where material flows with diffusion occur, it may be possible to propose different definitions and we shall in fact discuss a different definition for the heat transfer, which we call here $d \bar{Q}$ (cf. $\left.\left.{ }^{2}\right)^{4}\right)$ ), namely

$$
d U=d \bar{Q}-p d V+\sum_{k=1}^{n} h_{k} d_{c} M_{k},
$$

where $h_{k}=u_{k}+p v_{k}$ is the partial specific enthalpy; $v_{k}$ is the partial specific volume. $d_{e} M_{k}$ is the change of mass of the component $k$, as far as it is caused by mass transfer to or from the system 
("external change"). In addition there may exist a mass change $d_{i} M_{k}$ of component $k$ caused by a chemical reaction ("internal change"). We remark that (9) is the same as (3) as far as only mass transfer occurs without diffusion phenomena; we then have $d_{c} M_{k}=c_{k} d M$ (with the concentration $c_{k}=M_{k} / M$ ) and (9) becomes

$$
d U=d \bar{Q}-p d V+\sum_{k=1}^{n} c_{k} h_{k} d M=d \bar{Q}-p d V+h d M ;
$$

therefore $d \bar{Q}=d Q$ for such changes, hence (9) is the same as (3) in the special case where it is a physical law, but (9) differs from (3) in the general case where it serves as a definition of $d \bar{Q}$ (with diffusion).

§3. The definitions of $d Q$ and $d \bar{Q}$ and shifts $\varepsilon_{k}$ of the energy zero points. We now discuss the consequences of a shift $\varepsilon_{k}$ of the energy zero's of the components. Before doing this, we remark that if a chemical reaction is possible among the components a relation exists which the constants $\varepsilon_{k}$ must satisfy: to derive it, we consider the first law (1) for a change in which the system exchanges no matter with the surroundings, while the volume is constant. Then $d U=d Q$ is a directly measurable quantity, in which no arbitrary constant is left. According (7) we have

$$
d U=\Sigma_{k=1}^{n}\left(u_{k} d_{i} M_{k}+M_{k} d u_{k}\right) .
$$

For a chemical reaction $d_{i} M_{k}$ is proportional to the "stoechiometric coefficient" $v_{k}$

$$
d_{i} M_{k} / v_{k}=\text { independent of } k \text {. }
$$

(In chemistry one divides $v_{k}$ by the molar mass of $k$ to obtain numbers proportional to the traditional integral stoechiometric numbers) If we also write down (11), after shifting the energy zero's we get

$$
d \tilde{U}=\sum_{k=1}^{n}\left(\tilde{u}_{k} d_{i} M_{k}+M_{k} d \tilde{u}_{k}\right) .
$$

We now have $d U=d \tilde{U}=d Q$, because an energy change of a closed system is completely defined. Further $d u_{k}=d \tilde{u}_{k}$ (cf. (6)). Hence it follows from (11) and (13)

or with (6) and (12)

$$
\begin{gathered}
\sum_{k=1}^{n}\left(\tilde{u}_{k}-u_{k}\right) d_{i} M_{k}=0, \\
\Sigma_{k=1}^{n} \varepsilon_{k} \nu_{k}=0 .
\end{gathered}
$$

This is the relation between the $\varepsilon_{k}$ 's, which we sought.

We now consider the shifts in $d Q$ and $d \bar{Q}$ which result from the 
shifts $\varepsilon_{k}$ for an open system (mass transfer to or from the surroundings). We get, using (15)

$$
d \tilde{U}-d U=\Sigma_{k} \varepsilon_{k} d M_{k}=\Sigma_{k} \varepsilon_{k}\left(d_{i} M_{k}+d_{e} M_{k}\right)=\Sigma_{k}, \varepsilon_{k} d_{e} M_{k},
$$

further

$$
\begin{gathered}
\tilde{h} d M-h d M=\Sigma_{k} \varepsilon_{k} c_{k} d M, \\
\Sigma_{k}\left(\tilde{h}_{k} d_{e} M_{k}-h_{k} d_{e} M_{k}\right)=\Sigma_{k} \varepsilon_{k} d_{e} M_{k} .
\end{gathered}
$$

Hence we get for $d Q$ and $d \bar{Q}$

$$
\begin{aligned}
& d \tilde{Q}-d Q=\Sigma_{k} \varepsilon_{k}\left(d_{e} M_{k}-c_{k} d M\right), \\
& d \tilde{\bar{Q}}-d \bar{Q}=\Sigma_{k} \varepsilon_{k}\left(d_{e} M_{k}-d_{e} M_{k}\right)=0,
\end{aligned}
$$

so that $d \bar{Q}$ is not shifted, while $d Q$ is generally shifted if the energy zero's are changed.

The different definitions (3) and (9) may now be interpreted in the following way, where we include microscopic considerations in the discussion. The internal energy $U$ of a system consists of potential and kinetic energy of the particles. The potential energy (of the particles in the molecule, atom or nucleus) has always arbitrary constants undefined, whereas the kinetic energy has a well-determined zero-point. For a closed system, $d Q$ from (1) gives a change in energy caused by a transfer of microscopic kinetic energy amongst the molecules. (3) and (9) can be interpreted as different ways of splitting the change of internal energy in several parts. Since $d \bar{Q}$ in (9) is not affected by a change in the energy zero it will be a change of microscopic kinetic energy, while $d Q$ in (3) includes also a change in potential energy of the microscopic particles. However, a change $h_{k} d_{e} M_{k}$ in (9) is a change of energy which comprises potential as well as kinetic energy (internal energies will consist partly of kinetic energy, e. g., the kinetic energy of translation or of the vibration of the atoms in a molecule). The advantage of (9) over (3) is that $d \bar{Q}$ is only a change in microscopic kinetic energy; however, it is not certain that one could not find still other ways of splitting $d U$, where a heat transfer $d Q^{\prime}$ would be defined which would also be a change of microscopic kinetic energy only. (9) and (3) are both possible definitions; it may only be that one definition is more convenient than the other in certain applications (Some statements on the different definitions $d Q$ and $d \bar{Q}$ were already made in $\left.{ }^{4}\right)$ ). 
$\S 4$. The first law formulated with specific quantities. For nonuniform systems the physical laws must be stated as differential equations containing specific quantities, i.e., per unit mass. We can write (3) and (9) with specific quantities. We put

$$
\left.\begin{array}{rl}
d Q & =M d q ; d \bar{Q}=M d \bar{q} ; \\
U & =M u ; V=M v ; c_{k}=M_{k} / M ; \\
d_{i} M_{k} & =M d_{i} c_{k} ; d c_{k}=d_{e} c_{k}+d_{i} c_{k} ; d M_{k}=d_{i} M_{k}+d_{c} M_{k},
\end{array}\right\}
$$

hence $d_{e} M_{k}=c_{k} d M+M d_{e} c_{k}$.

We then get instead of (3)

$$
d u=d q-p d v,
$$

and instead of (9)

$$
d u=d \bar{q}-p d v+\sum_{k=1}^{n} h_{k} d_{e} c_{k} .
$$

Though (22) may seem simpler than (23) in this formulation, it must be remembered that $d q$ changes for a shift of the energy zero's, whilst $d \bar{q}$ doesn't.

$\S 5$. The fundamental laws for irreversible thermodynamics. We formulate the four fundamental laws, which are the basis for the thermodynamics of irreversible processes in continuous systems $\left.{ }^{1}\right){ }^{3}$ ) (we follow the notation of ${ }^{3}$ ) and take a continuous system in which heat conduction, diffusion and one chemical reaction may occur; for convenience, however, we exclude viscosity terms).

I. The law of conservation of mass

$$
\partial \varrho_{k} / \partial t=-\operatorname{div} \varrho_{k} \mathbf{v}_{k}+v_{k} J_{c} .
$$

This can also be written as

$$
\varrho d c_{k} / d t=-\operatorname{div} \mathbf{J}_{k}+\nu_{k} J_{c^{\circ}}
$$

We can put

so, that

$$
\begin{gathered}
\varrho d_{c} c_{k} / d t=-\operatorname{div} \mathbf{J}_{k}, \\
\varrho d_{i} c_{k} / d t=v_{k} J_{c},
\end{gathered}
$$

$$
d c_{k}=d_{c} c_{k}+d_{i} c_{k}
$$


II. The equation of motion

$$
\varrho d \mathbf{v} / d t=-\operatorname{grad} p+\Sigma_{k=1}^{n} . \mathbf{F}_{k} \varrho_{k} .
$$

III. The en ergy equation expresses that the energy change $\partial e_{v} / \partial t$ of the total energy $e_{v}$ per unit of volume is equal to the divergence of the total energy flow $\mathbf{J}_{\epsilon}$ plus a source term caused by external forces acting on the components

$$
\partial e_{v} / \partial t=-\operatorname{div} \mathbf{J}_{\epsilon}+\sum_{k=1}^{n} \mathbf{F}_{k} \cdot \mathbf{v}_{k} \varrho_{k} .
$$

The total energy $e_{v}$ consists of internal energy and kinetic energy; we may also take it per unit of mass; we then call it $e$ and put

$$
e_{v}=\varrho e .
$$

We have

$$
e=u+\frac{1}{2} \mathbf{v}^{2}
$$

where $u$ is the internal energy per unit of mass and $\frac{1}{2} \mathbf{v}^{2}$ the bulk kinetic energy per unit of mass. We can think of $u, \frac{1}{2} \mathbf{v}^{2}$ and $e$ as measurable quantities: $u$ is a function of the state variables $p, T$ and the concentrations (and one can determine this fuction according to $\S 1)$. $\mathbf{v}$ is the bulk velocity of a volume element. $\mathbf{J}_{e}$ can be measured if we measure the change of energy in systems $S_{1}, S_{2}, \ldots$ each of which has part of its boundary in common with the element of the system which is considered, while their other walls are insulating (Fig. 3).

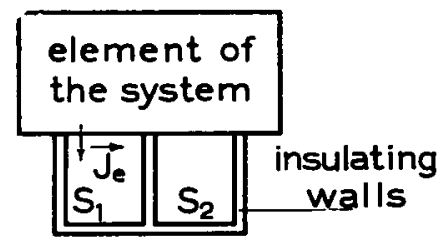

Fig. 3. In principle it is possible to give a method to determine the total energy flow $\mathbf{J}_{c}$ by measuring the energies in separate volumes.

We now introduce heat flows $\mathbf{J}_{q}$ and $\mathbf{J}_{\bar{q}}$ by definitions in a way that is analogous to the introduction of $d Q$ and $d \bar{Q}$ in $\S 2$.

$$
\begin{gathered}
\mathbf{J}_{c}=\mathbf{J}_{q}+\left(\frac{1}{2} \mathbf{v}^{2}\right) \varrho \mathbf{v}+h \varrho \mathbf{v}, \\
\mathbf{J}_{\epsilon}=\mathbf{J}_{\bar{q}}+\left(\frac{1}{2} \mathbf{v}^{2}\right) \varrho \mathbf{v}+\Sigma_{k} h_{k} \varrho_{k} \mathbf{v}_{k} .
\end{gathered}
$$

If diffusion phenomena occur, all quantities can be measured except $\mathbf{J}_{q}$ or $\mathbf{J}_{\bar{q}}$, so that these equations are no longer physical laws but 
definitions of $\mathbf{J}_{q}$ and $\mathbf{J}_{\bar{q}}$. (Without diffusion phenomena (33) and (34) become the same; $\mathbf{J}_{q}=\mathbf{J}_{\bar{q}}$ is then directly measurable and the equations express the energy law, together with (30)).

We now show that (33) and (34) are analogous to (3) and (9) (together with (30)). As a matter of fact, if we introduce

$$
\begin{aligned}
& \varrho d q / d t=-\operatorname{div} \mathbf{J}_{q}, \\
& \varrho d \bar{q} / d t=-\operatorname{div} \mathbf{J}_{\bar{q}},
\end{aligned}
$$

we get from $(26), \ldots,(36)$

$$
d u / d t=d q / d t-p d v / d t+(1 / \varrho) \Sigma_{k} \mathbf{F}_{k} . \mathbf{J}_{k},
$$

and $\quad d u / d t=d \bar{q} / d t-p d v / d t+\Sigma_{k} h_{k} d_{i} c_{k}+(1 / \varrho) \Sigma_{k} \mathbf{F}_{k} . \mathbf{J}_{k}$,

which are the same as (22) and (23) respectively. The last terms in (37) and (38) have no counterpart in (22) and (23), because in sections $\S \S 2-4$, we had supposed that no external forces $\mathbf{F}_{k}$ existed.

IV. The second law of thermodynamics is

$$
T d s / d t=d u / d t+p d v / d t-\Sigma_{k} \mu_{k} d c_{k} / d t .
$$

From (25), (37), resp. (38) and (39) we get for the entropy balance

$$
\varrho d s / d t=-\operatorname{div} \mathbf{J}_{s}+\sigma .
$$

where the entropy flow $\mathbf{J}_{s}$ is equal to

$$
\mathbf{J}_{s}=\left(\mathbf{J}_{q}-\Sigma_{k} \mu_{k} \mathbf{J}_{k}\right) / T,
$$

or (cf. also ${ }^{1}$ ) p. 97)

$$
\mathbf{J}_{s}=\mathbf{J}_{\bar{q}} / T+\Sigma_{k} s_{k} \mathbf{J}_{k} \text {. }
$$

The choice of $\mathbf{J}_{\bar{q}}$ seems to be more natural than $\mathbf{J}_{q}$, if we look at these equations for $\mathbf{J}_{s}$. In (42) the second part has the appearance of a convection term of entropy, while this is not true for (41).

The entropy production $\sigma$ is given either by

$$
\sigma=\left(\mathbf{J}_{q} \cdot \mathbf{X}_{q}+\Sigma_{k} \mathbf{J}_{k} \cdot \mathbf{X}_{k}+A \mathbf{J}_{c}\right) / T,
$$

with

$$
\begin{aligned}
& \mathbf{X}_{q}=-(\operatorname{grad} T) / T, \\
& \mathbf{X}_{k}=\mathbf{F}_{k}-\operatorname{grad}\left(\mu_{k} / T\right), \quad A=-\dot{\Sigma}_{k} \mu_{k} \nu_{k},
\end{aligned}
$$

or by

$$
\left.\sigma=\left(\mathbf{J}_{\bar{q}} \cdot \mathbf{X}_{\bar{q}}+\Sigma_{k} \mathbf{J}_{k} \cdot \mathbf{X}_{k}^{\prime}+A\right\rfloor_{c}\right) / T,
$$


with $\quad \mathbf{X}_{\bar{q}}=-(\operatorname{grad} T) / T$,

$\mathbf{X}_{k}^{\prime}=\mathbf{F}_{k}-\operatorname{grad} \mu_{k}-s_{k} \operatorname{grad} T=\mathbf{F}_{k}-\left(\operatorname{grad} \mu_{k}\right)_{T}$.

We can also write the change of entropy in a form analogous to (24) and (30)

$$
\partial s_{v} / \partial t=-\operatorname{div}\left(\mathbf{J}_{s}+s_{v} \mathbf{v}\right)+\sigma
$$

where $s_{v}=\varrho s$ is the entropy per unit volume.

Reccived 28-8-52.

\section{REFERENCES}

1) Prigogine, I., Étude thermodynamique des phénomènes irréversibles, Thesis, Brussels, 1947.

2) Gillespie, L. J., and Coe, J. R., J. chem. Phys, 1 (1933) 103.

3) de Groot, S. R., Thermodynamics of irreversible processes, Nortl Holland Publishing Company, Amsterdam and Interscience Publishers, New York, 1951.

4) Tolhoek, H. A., Dissertation, Utrecht, 1951, Thesis XII. 